\author{
Повышение скорости работы \\ многоразрядного двоичного умножителя \\ А.Н. Якунин ${ }^{1}$, Аунг Мьо Сан ${ }^{2}$ \\ Национальный исследовательский университет «МИЭТ», г. Москва \\ 1'yakunin.alexey@gmail.com; ${ }^{2}$ aungmyosan61028@gmail.com
}

\begin{abstract}
Аннотация - В данной статье рассмотрены два типа многоразрядных двоичных умножителей, реализующих арифметическое умножение двух положительных чисел с фиксированной точкой: ведический умножитель и модифицированный древовидный умножитель. В работе проведено моделирование обеих архитектур для $8 \times 8$, $16 \times 16,32 \times 32$ - разрядных двоичных умножителей в среде САПР Quartus II на базе ПЛИС Altera EP2SGX30DF780C3 семейства Stratix-II-GX. Выполнен их сравнительный анализ по аппаратным и временным затратам. По результату сравнений при реализации $32 \times 32$ разрядного двоичного умножителя умножитель, предложенный в этой работе, даёт выигрыш по скорости до $23 \%$ по сравнению с ведическим умножителем. Аппаратные затраты в предложенном умножителе снижаются на $22 \%$ по сравнению с ведическим умножителем. Кроме того, предложенную структуру можно масштабировать на большее количество разрядов, например $64 \times 64,128 \times 128,256 \times 256$ битов и т.д.
\end{abstract}

Ключевые слова - двоичный умножитель; полусумматор; полный сумматор; параллельно-префиксный сумматор (ППС); сумматор с запоминанием переноса (СЗП); ведический умножитель (BУ); ALUTs; время задержки.

\section{I. ВВЕДЕНИЕ}

На сегодняшний день в цифровой схемотехнике большое внимание уделяется быстродействию работы устройств, реализующих арифметические операции, такие как сложение, вычитание, умножение, деление и т.д. [1]. Аппаратная реализация арифметических операций над двоичными числами является важным архитектурным элементом в микропроцессорах, цифровых сигнальных процессорах, математических сопроцессорах и других приложений [2]. Многие арифметические операции опираются на сложение, поэтому имея аппаратную структуру сумматора становится возможным реализовать умножение путём повторного сложения, вычитание путём логического отрицания одного операнда и деление путём повторного вычитания [3]. При схемной реализации выполнения арифметических операций чем меньше время задержки поступления данных на выход действующего устройства, тем выше его скорость работы. Одним из способов сокращения временных затрат является переход к параллельной архитектуре действующего устройства, в которой применяются базовые логические элементы: «НЕ», «И», «ИЛИ», «ИНЕ», «ИЛИ-НЕ» и т.Д.

Умножитель - это логический комбинационный узел, выполняющий операцию умножения двух двоичных чисел. Умножители входят в состав более сложных цифровых устройств, например арифметикологических устройств (АЛУ). Поэтому аппаратная реализация эффективного умножителя необходима для повышения быстродействия АЛУ и, следовательно, процессора в целом.

В последнее десятилетие было изучено и разработано множество структур многоразрядных двоичных умножителей, построенных на основе различных методов ускорения умножения. По принципу суммирования частичных произведений, которые формируются путём умножения отдельных разрядов множителя на всё множимое, двоичные умножители подразделяются на матричные и древовидные. В обоих структурах для суммирования частичных произведений широко применяются массивы взаимосвязанных комбинационных двоичных сумматоров. В реализации матричных умножителей двоичные сумматоры формируются в виде матрицы, а в древовидных - в виде дерева. Существующими вариантами матричных структур являются умножитель Брауна, умножитель Бо-були и умножитель Пезариса [4]. В них операция умножения сводится к параллельному формированию битов из n-разрядных частичных произведений $\mathrm{c}$ последующим их суммированием с помощью матриц сумматоров.

Сокращение времени выполнения суммирования частичных произведений в матричных умножителях реализуется в схемах, построенных по древовидной структуре. Если в матричных умножителях для суммирования $\mathrm{n}$ частичных произведений требуется $\mathrm{n}$ строк сумматоров, то в древовидных схемах количество каскадов сумматоров пропорционально $\log _{2} n$. Это приводит к сокращению времени вычисления суммирования частичных произведений. Однако при аппаратной реализации таких умножителей требуются дополнительные связи для объединения разрядов, имеющих одинаковых вес. Изза этого их аппаратные затраты выше. К древовидным умножителям относятся умножитель со схемой 
перевернутого дерева [4], умножитель Уоллеса [4, 5], умножитель Дадда $[4,6]$.

В настоящее время к умножителям, реализующим аппаратные методы ускорения, относятся ведические умножители. Такой тип умножителя построен на основе ведической математики. Подробности ведической математики и реализации ведического умножителя рассмотрены в работе [7]. В ней проведено сравнение аппаратных и временных затрат ведического умножителя с другими умножителями, такими как матричный умножитель, умножитель с деревом Уоллеса и умножитель Бута. В результате сравнения в этой работе выявлено, что ведический умножитель обладает минимальной задержкой по сравнению с другими умножителями.

В данной работе рассмотрены два типа многоразрядных двоичных умножителей: ведический умножитель и модифицированный древовидный умножитель. Моделирование умножителей обеих схем для разрядностей $8 \times 8,16 \times 16$ и $32 \times 32$ проведено в среде САПР Quartus II на базе ПЛИС Altera EP2SGX30DF780C3 семейства Stratix-II-GX и выполнен их сравнительный анализ. Для оценки аппаратных и временных затрат обоих умножителей определены следующие параметры: аппаратная сложность по количеству логических блоков ПЛИС (единицей измерения в Quartus II являются так называемые ALUTs [8]) и максимальное время задержки. Достоверность выполнения операции умножения двоичных чисел подтверждена временными диаграммами результатов моделирования.

При аппаратной реализации многоразрядных двоичных умножителей сумматоры играют важную роль для выполнения суммирования частичных произведений. Поэтому прежде всего в работе проанализированы полусумматор и полный сумматор для сложения двух одноразрядных двоичных чисел. Затем для сложения двух многоразрядных двоичных чисел реализован параллельно-префиксный сумматор.

\section{II. АППАРАТНАЯ РЕАЛИЗАЦИЯ ДВОИЧНЫХ СУММАТОРОВ}

\section{А. Полусумматор и полный сумматор}

Одноразрядные сумматоры выполняют сложение двух одноразрядных двоичных слагаемых. По числу входов и выходов одноразрядные двоичные сумматоры можно разделить на полусумматор и полный сумматор. Полусумматор имеет два входа ( $A$ и $B$ ) и два выхода $\left(S\right.$ и $\left.C_{\text {out }}\right) . S$ - это сумма $A$ и $B$. Если $A$ и $B$ равны 1 , то выход $S$ должен стать равным 2, но такое число не может быть представлено в виде одного двоичного разряда. В этом случае результат указывается вместе с переносом $C_{\text {out }}$ в следующий разряд. Таблица истинности отражает работу полусумматора, а логические уравнения и схемная реализация полусумматора показаны на pис.1a. Буквы HA (Half Adder) используются для обозначения полного сумматора.

Полный сумматор имеет три входа: $A$ и $B$ - разряды слагаемых и $C_{i n}-$ перенос из предыдущего (младшего) разряда; и два выхода: $S$ - сумма по данному разряду и $C_{\text {out }}-$ перенос в следующий разряд.

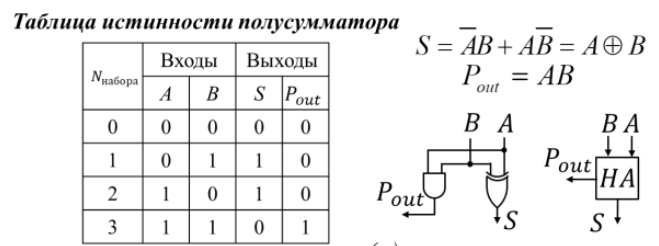

(a)

Таблица истинности полного сумматора

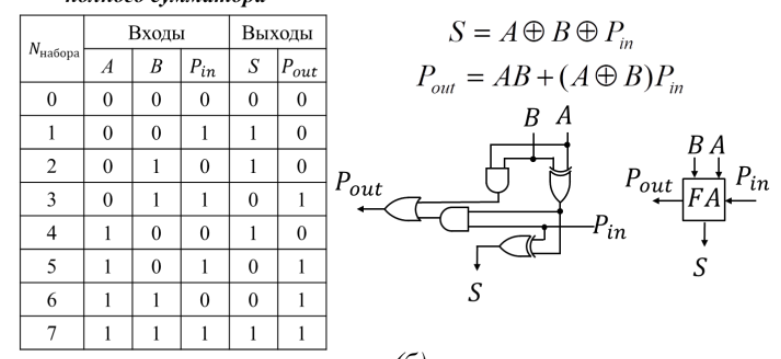

(б)

Рис. 1. $\boldsymbol{a}$ - полусумматор; $\sigma$ - полный сумматор

Работу полного сумматора отражает таблица истинности, а схемная реализация и логические уравнения полного сумматора показаны на рис.1б. Буквы FA (Full Adder) используются для обозначения полного сумматора.

\section{В. Параллельно-префиксный сумматор (ППС)}

Сложение двух многоразрядных двоичных чисел может быть представлено схемой, состоящей из трёх каскадов (рис. 2) [9]: каскада предвычисления, каскада формирования параллельно-префиксного дерева и каскада формирования результата. На первом каскаде осуществляется предварительное вычисление битов $g_{i}=A_{i} B_{i}$, генерирующих сигнал переноса, битов $h_{i}=A_{i} \oplus B_{i}$, передающих сигнал переноса для любого входных операндов, $0 \leq i \leq n-1$.

На втором каскаде группа сигналов генерации переноса $G_{i: k}$ и распространения переноса $H_{i: k}$ вычисляется для каждого бита $0 \leq i \leq n-1$ по следующим логическим уравнениям:

$$
\begin{gathered}
G_{[i: k]}=\left\{\begin{array}{ll}
g_{i} & \text { если } i=k \\
G_{[i: j]}+H_{[i: j]} \cdot G_{[j-1: k]} ; & \text { в противном случае }
\end{array}\right\} \\
H_{[i: k]}=\left\{\begin{array}{ll}
h_{i} ; & \text { если } i=k \\
H_{[i: j]} \cdot H_{[j-1: k]} ; & \text { в противном случае }
\end{array}\right\}
\end{gathered}
$$

Используя уравнения (1) и (2), и $G_{i: k}$ и $H_{i: k}$ используют входы из верхней части, охватывающей биты $i: j$, и из нижней части, охватывающей биты $j-1: k$. Затем эти части объединяются для формирования дерева сигналов генерации и 
распространения всего переноса, охватывающего биты $i: k$.

Используя сигналы $G_{i: k}$ из второго каскада, на последнем каскаде вычисляются выходные биты суммы $S_{i}$ и бит выходного переноса по следующим формулам:

$$
\begin{gathered}
P_{i}=G_{[i: k]} \\
S_{i}=h_{i} \oplus P_{i-1}
\end{gathered}
$$

где $P_{-1}-$ входной сигнал $P_{i n}=0$.

На рис. 2 представлены 8-разрядный ППС и реализация используемых базовых схематичных узлов. Введены схематичные узлы: чёрный узел, белый узел, прямоугольник и треугольник, применяемые для большей наглядности при построении ППС.

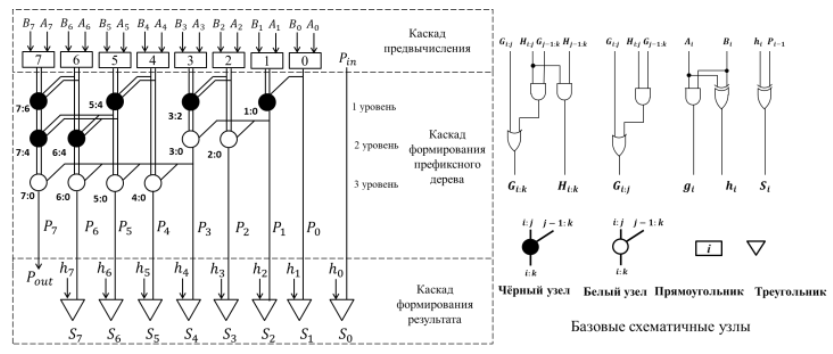

Рис. 2. 8-разрядный ППС

В данной схеме сначала вычисляются $g_{i}$ и $h_{i}$ для пар разрядов первого каскада, далее для блоков из 4-х разрядов, затем для узлов из каскада формирования префиксного дерева, пока сигнал $P_{i}$ не будет известен для каждого разряда. После этого результат $S_{i}$ операции сложения вычисляется вместе с $P_{i}$ в последнем каскаде по приведённым выше формулам (3), (4). Время задержки такого ППС пропорционально количеству уровней на каскаде формирования префиксного дерева. На этом каскаде количество уровней соответствует $\log _{2} n$. С наращиванием разрядности входных операндов на рис. 3 показана схема параллельно-префиксного сумматора для 16-разрядных операндов.

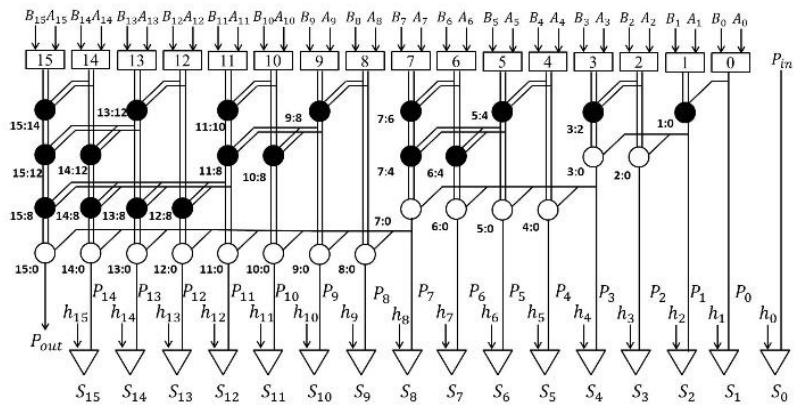

Рис. 3. 16-разрядный параллельно-префиксный сумматор

\section{C. Сумматор с запоминанием переноса (СЗП)}

Одноразрядный сумматор с запоминанием переноса [10] представляет собой одноразрядный полный сумматор с входом $C_{i n}$, переименованным в $C$, и выход $C_{\text {out }}$ переименованным в $P$. Идея состоит в том, чтобы входные складываемые 3 числа $A, B$ и $C$ преобразовать в два таких числа $P$ и $S$, чтобы $A+B+C=P+S$. На рис. $4 a$ показано, как $n$ сумматоров с запоминанием переноса расположены так, чтобы при сложении трёх $n$-разрядных двоичных чисел $A, B$ и $C$ сформировать результат в виде $P$ и $S$. Схема параллельного суммирования четырёх входных операндов с $n$-разрядами приведена на рис. 46. Чтобы получить окончательный результат суммирования, необходимо сложить $P$ и $S$ по каждому разряду. Все полные сумматоры в трёхоперандном СЗП с $n$ разрядами независимы друг от друга, поэтому вся схема имеет задержку только полного сумматора.

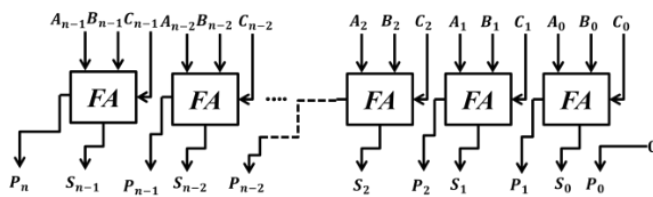

(a)

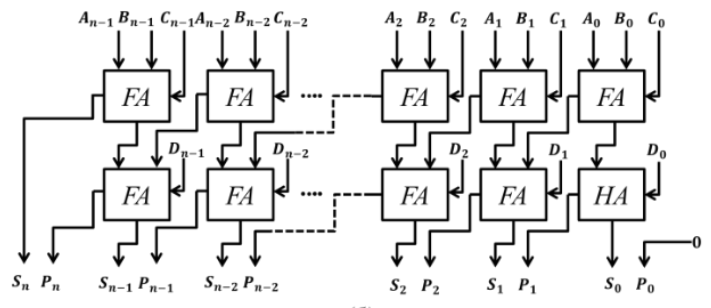

(б)

Рис. 4. $a$ - Трёхоперандный СЗП с $n$-разрядами; $\sigma$ Четырёхоперандный СЗП с $\boldsymbol{n}$ - разрядами

В схеме трёхоперандного СЗП с $n$ - разрядами видно, что в нулевом разряде первый полный сумматор выдаёт сумму трёх операндов $S_{0}$ и перенос в следующий разряд $P_{1}$, а не $P_{0}$. Поэтому при сложении в нулевом разряде значение переноса $P_{0}=0$. Такая же концепция может быть применена к схеме четырёхоперандного СЗП с $n-$ разрядами. Следует обратить внимание на то, что в показанных схемах желаемый полный сумматор может быть заменён на полусумматор без третьего операнда $C$.

\section{III. АППАРАТНАЯ РЕАЛИЗАЦИЯ ВЕДИЧЕСКОГО УМНОЖИТЕЛЯ}

В данном разделе рассмотрим алгоритм умножения десятичных и двоичных чисел на основе ведической математики. Чтобы проиллюстрировать этот алгоритм, возьмём умножение двух десятичных чисел $(\mathrm{A}=25$, $\mathrm{B}=76)$ и двух двоичных чисел $(\mathrm{A}=11$ и $\mathrm{B}=11)$ по методу Урдхва Тирякхьяма [7], как показано на рис. 1. 


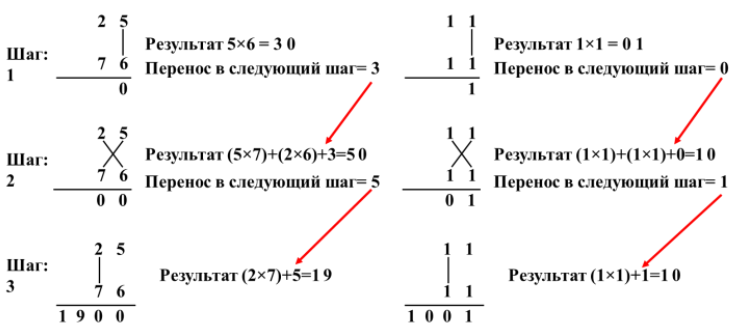

Рис. 5. Алгоритм умножения двух десятичных и двоичных чисел на основе ведической математике

Из рис. 6 видно, что сначала нужно взять две цифры ( $A_{0}$ и $\left.B_{0}\right)$ в нулевом разряде как от множителя, так и от множимого. Затем умножить их между собой и получить первый бит результата $R_{0}$ и перенос $P_{1}$, поступающий на следующий шаг. На втором шаге умножаем цифры $\left(A_{0} \times B_{1}\right)$ и $\left(A_{1} \times B_{0}\right)$ путём умножения крест-на-крест и выполняем сложение $\left(A_{0} \times B_{1}\right)+\left(A_{1} \times B_{0}\right)+P_{1}$. Получаем второй бит ответа $R_{1}$ и перенос $P_{2}$ на следующий шаг. На последнем шаге умножаем $A_{1}$ на $B_{1}$ и выполняем сложение $\left(A_{1} \times B_{1}\right)+P_{2}$. Получаем $S_{2}$ и $P_{3}$. Сумма $S_{2}$ является третьим соответствующим битом, а перенос $P_{3}$ становится четвертым битом конечного ответа.

По этому методу можно создать схему $(2 \times 2)$ ведического умножителя (ВУ) с использованием четырех двухвходовых логических элемента «И» и двух полусумматоров. Схема $(2 \times 2)$ ведического умножителя показана на рис. 6.

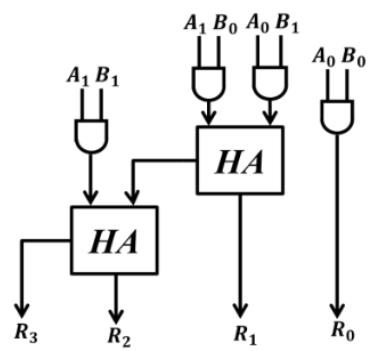

Рис. 6. $(2 \times 2)$ ведический умножитель

Описанный метод может быть расширен на большее количество входных операндов, но для этого потребуются дополнительные аппаратные затраты. Ведический умножитель $(4 \times 4)$ разряда создается с использованием четырёх блоков $(2 \times 2)$ умножения. Кроме этого для сложения промежуточных результатов из каждого $(2 \times 2)$ умножителя требуются сумматоры с запоминанием переноса, а также для генерации окончательных результатов необходимо применять параллельно-префиксный сумматор.

Для наращивания разрядности входных операндов ведического умножителя на рис. 7 и рис. 8 приведены схемы $(4 \times 4),(8 \times 8),(16 \times 16)$ и $(31 \times 31)$ ведических умножителей.

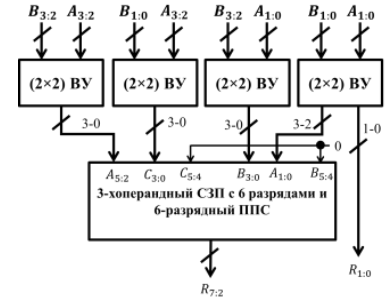

(a)

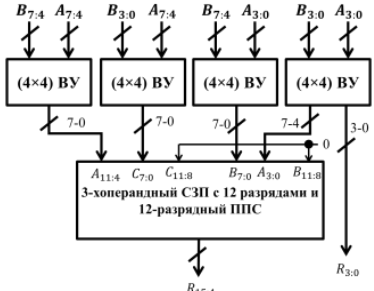

(б)
Pис. 7.a - $(4 \times 4)$ ведический умножитель; $\boldsymbol{\sigma}$ - $(8 \times 8)$ ведический умножитель

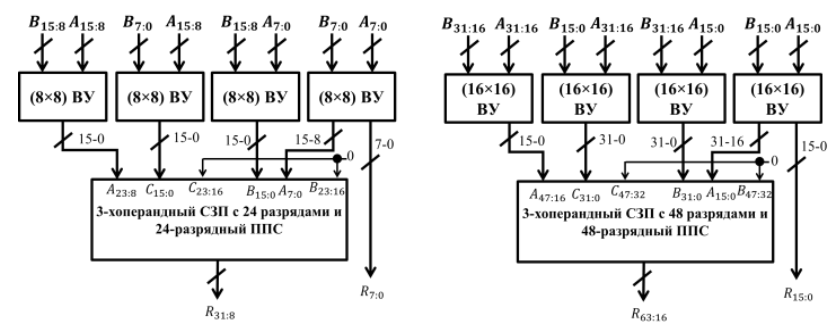

Рис. 8. $\boldsymbol{a}$ - $(16 \times 16)$ ведический умножитель; б - $(31 \times 31)$ ведический умножитель

А. Реализация модифицированного древовидного умножителя

В рамках данной работы авторами предложена схема модифицированного умножителя, в котором используется древовидная структура. Для реализации этой схемы на рис. 9 проведена точечная структура умножения двух 16×16-разрядных двоичных чисел, которая используется для реализации модифицированного умножителя.

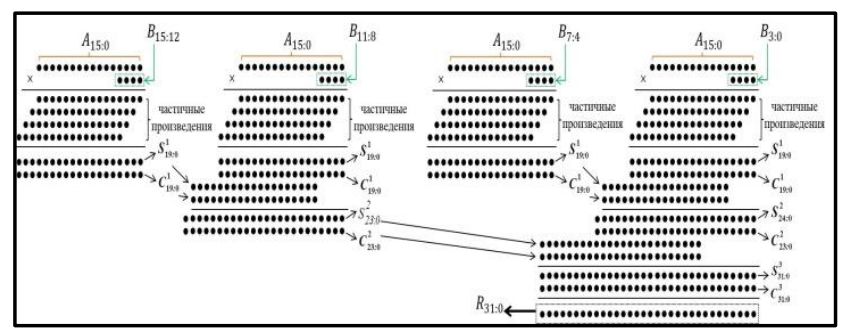

Рис. 9. Точечная структура для выполнения 16×16разрядного умножения

Принцип работы данного умножителя виден из рисунка и состоит из следующих шагов:

1-й шаг: 16-разрядный множитель В15:0 (второй операнд) разбивается на 4 группы по четыре бита: В15:12, В11:8, В7:4 и В3:0.

2-й шаг: Умножается 16-разрядное множимое А (первый операнд) на каждую группу множителя В, и получаются 4 частичные произведения для каждой группы.

3-й шаг: Выполняется суммирование частичных произведений из каждой группы и выдаются значения $S_{18: 0}^{l}$ и $P_{18: 0}^{l}$. 
4-й шаг: Приписываются значения $S_{18: 0}^{1}$ и $P_{18: 0}^{l}$ из второй и четвёртой группы к значениям $S_{18: 0}^{1}$ и $P_{18: 0}^{l}$ из третьей и первой группы со сдвигом на 4 бита. Затем они суммируются, и выдаются значения $S_{23: 0}^{2}$ и $P_{23: 0}^{2}$ для первой и третьей группы.

4-й шаг: Приписываются значения $S_{23: 0}^{2}$ и $P_{230}^{2}$ из третьей группы к значениям $S_{23: 0}^{2}$ и $P_{230}^{2}$ из первой группы со сдвигом на 8 битов. Затем они суммируются, и выдаются значения $S_{31: 0}^{3}$ и $P_{31: 0}^{3}$ для первой группы.

5-й шаг: Наконец складываются $S_{31: 0}^{3}$ и $P_{310}^{3}$ для первой группы, и получается $2 \mathrm{n}$ - разрядный результат.

На рис. 10 представлена схема $(16 \times 4)$-блока, состоящего из матрицы логических элементов «И» $(16 \times 4)$ четырёх-операндный СЗП для сложения частичных произведений.

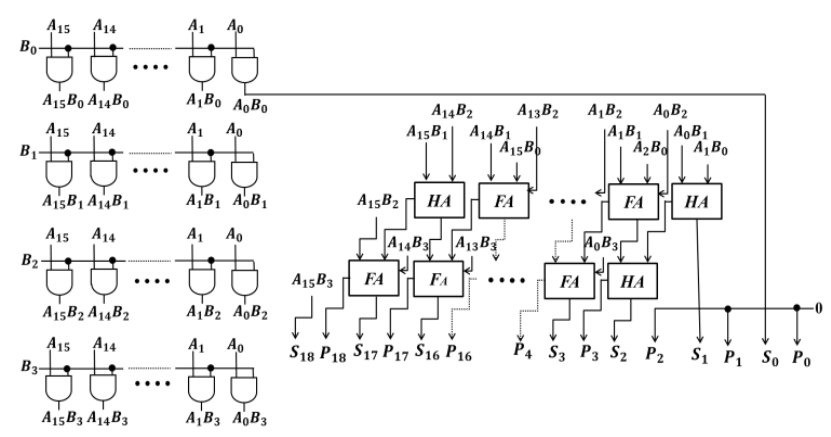

Рис. 10. Схема $(16 \times 4)$-блока

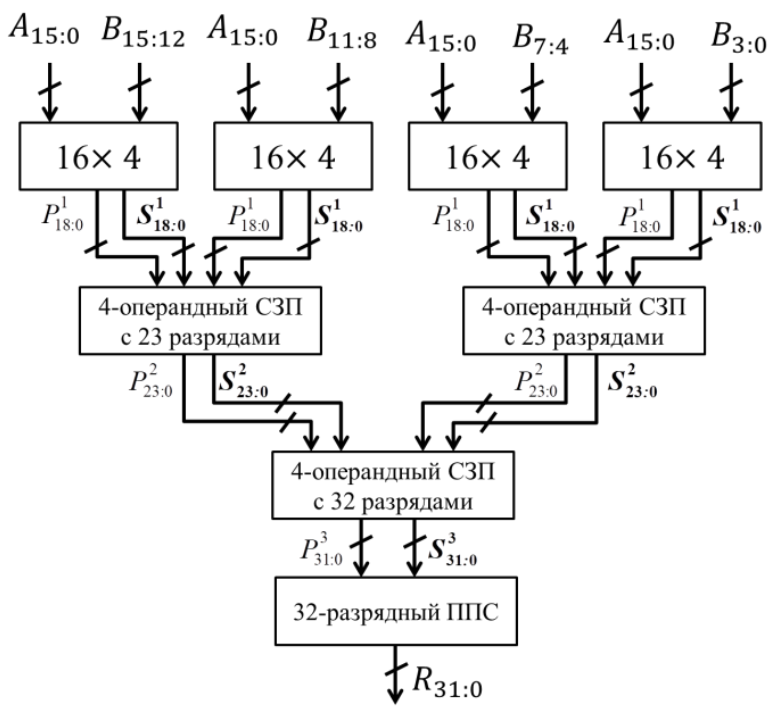

Рис. 11. 16×16-разрядный модифицированный умножителя

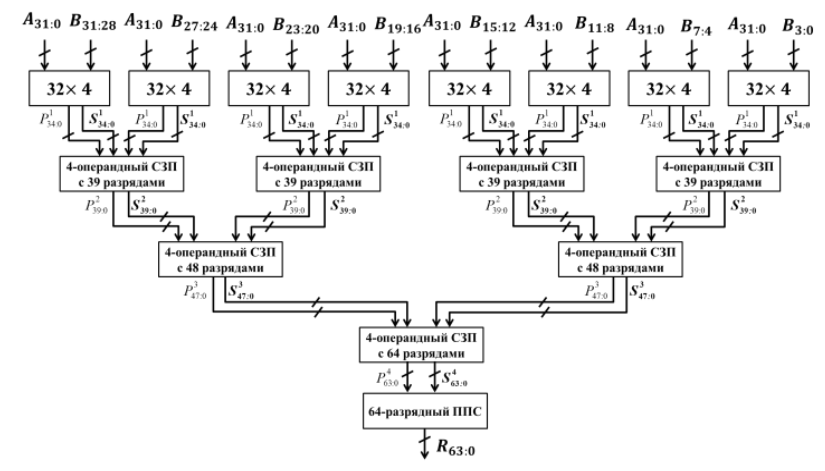

Рис. 12. 32×32-разрядный модифицированный умножителя

Используя рассмотренный принцип на рис. 11 предложены 16×16-разрядный модифицированный умножитель, содержащий 4 (16×4)-блоков, три 4операндных СЗП и 32-разрядный ППС. На рис. 12 представлен $32 \times 32$-разрядный модифицированный умножитель.

\section{IV. СРАВНИТЕЛЬНЫЙ АНАЛИЗ И РЕЗУЛЬТАТЫ МОДЕЛИРОВАНИЯ УМНОЖИТЕЛЕЙ}

Моделирование схем двух умножителей для выполнения $8 \times 8,16 \times 16$ и $32 \times 32$-разрядных двоичных чисел проведено в среде САПР Quartus II на базе ПЛИС Altera EP2SGX30DF780C3 семейства Stratix-II$G X$. Для оценки аппаратных и временных затрат определим значение комбинационных логических блоков ALUTS $\left(H_{\text {ALUTS }}\right)$ и максимальное время задержки $t_{\max }$ по результату моделирования схем умножителей. Значение $t_{\max }$ может быть получено из формулы: $t_{\max }=t_{\text {cell }}+t_{L}$; где, $t_{\text {cell }}$ - общая задержка логических элементов и $t_{L}$ - общая задержка их межсоединений. В таблице 1 приведены оценки аппаратных и временных затрат двух умножителей для вычисления $8 \times 8,16 \times 16,32 \times 32$-разрядных двоичных чисел, которые получены в результате моделирования.

Таблица 1

Оценки аппаратных и временных затрат двух двоичных умножителей

\begin{tabular}{|c|c|c|c|c|}
\hline \multirow{2}{*}{ разрядность } & \multicolumn{2}{|c|}{$\begin{array}{l}\text { Ведический } \\
\text { умножтель }\end{array}$} & \multicolumn{2}{|c|}{$\begin{array}{c}\text { Модифицированный } \\
\text { умножитель }\end{array}$} \\
\hline & $H_{A L U T}$ & $\begin{array}{l}t_{\max } \\
(H c)\end{array}$ & $H_{A L U T}$ & $\begin{array}{l}t_{\max } \\
(H C)\end{array}$ \\
\hline $8 \times 8$ & 151 & 15.0 & 119 & 14.9 \\
\hline $16 \times 16$ & 666 & 28.7 & 553 & 18.9 \\
\hline $32 \times 32$ & 2852 & 31.6 & 2201 & 24.3 \\
\hline
\end{tabular}

Анализ результатов моделирования показывает, что аппаратные затраты у модифицированного умножителя меньше, чем у ведического умножителя, в частности у $32 \times 32$-рязрядных умножителей эта 
разница равна 651 ALUTs (22\%). Кроме того, модифицированный умножитель даёт выигрыш в скорости до 23\% по сравнению с ведическим умножителем. Результаты показывают, что чем больше разрядность умножителей, тем больше процентная разность скорости работы и аппаратной сложности между ними.

Достоверность выполнения операции 32×32разрядного двоичного умножения подтверждена результатами моделирования (временными диаграммами), полученными в среде САПР Quartus II и представленными на рис. 13.

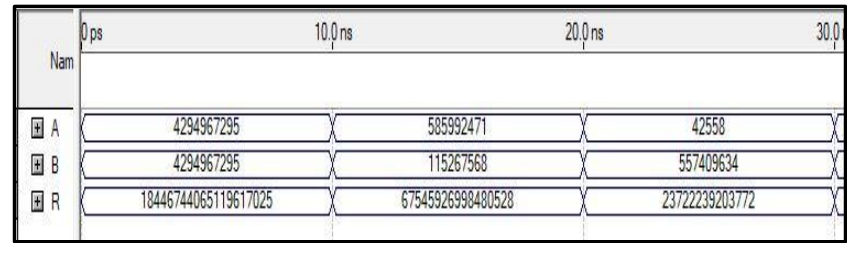

Рис. 13. Временная диаграмма результатов моделироания ( $32 \times 32)$-разрядного модифицированного умножителя

\section{V. ЗАКЛЮЧЕНИЕ}

В данной работе приведены схемные реализации полусумматора, полного сумматора, параллельнопрефиксного сумматора и мульти-операндного сумматора с запоминанием переноса для выполнения арифметического сложения двоичных чисел. Реализован ведический двоичный умножитель, Предложен модифицированный умножитель и проведён их сравнительный анализ. Результаты сравнения показали, что предложенный умножитель имеет лучшие параметры по аппаратным и временным затратам по сравнению с ведическим. Структуру умножителя можно масштабировать, спроектировав умножители с большим количеством разрядов, таких как 64, 128, 256 бит и т.д. Интересным направлением дальнейшей разработки является исследование возможности применения многоразрядного высокоскоростного умножителя для модулярной арифметики.

\section{ЛИТЕРАТУРА}

[1] Червяков Н.И., Ляхов П.А., Валуева М.В., Криволапова О.В. Сравнительный анализ аппаратной реализации сумматороа на FPGA // «Науки. Инновации. Технологии» Северо-Кавказский федеральный университет. 2016. № 4. С. 98.

[2] Акаш Кумар, Дипика Шарма. Анализ производительности различных типов сумматоров для высокоскоростного 32-битного умножения и накапливания. 2013. -V. 3. С. 1460.

[3] Проф. Рашми Рахул Кулкарни. Сравнение между различными сумматорами // Журнал IOSR СБИС и обработка сигналов. 11-12, 2015. V 5. С. 2319-4197.

[4] С. А. Орлов., Б. Я. Цилькер. Организация ЭВМ и системы. Учебник для вузов. 2-е издание, 2011. С. 192206.

[5] Bhupender Pratap Singh., Rakesh Kumar. Design and Implementatin 8-bit Wallace Tree Multiplier. International Journal of Advanced Research in Electrical Electronics and Instrumentation Engineering. Vol. 5, Issye 4, April 2016. P. 2307-2311.

[6] Palaniappan Ramanathan., Ponnisamy Thangapandian Vanathi., Sundeepkumar Agarwal. High Speed Multiplier Design Using Decomposition Logic. Serbian journal of electrical engineering. Vol. 6, No. 1, May 2009, 33-42.

[7] Abhijeet Kumar., Dilip Kumar., Siddhi. Hardware Implementation of $16 * 16$ bit Multiplier and Square using Vedic Mathematics. International Conference on Signal, Image and Video Processing (ICSIVP) 2012. P. 309-314.

[8] Altera Corporation. Stratix II Device Handbook, Volume 1 2005// URL: http://www.ece.iastate.edu/ zambreno/classes/ cpre583/documents/altera/stx2_sii5v1_01.pdf. P. 20-22.

[9] Дэвид Харрис. Таксономия параллельных префиксных сетей. Колледж Харви Мадд. IEEE 2013 / Р. 2213-2214.

[10] Рето Циммерманн. Архитектуры двоичного сумматора для клеточного СБИС и их синтеза. Швейцарский федеральный институт технологии, Цюрих, 1997. С. 34 39.

\title{
Increasing the Speed of a Multi-bit Binary Multiplier
}

\author{
A.N. Yakunin ${ }^{1}$, Aung Myo $\mathrm{San}^{2}$ \\ National Research University "MIET", Moscow \\ 1'yakunin.alexey@gmail.com; ${ }^{2}$ aungmyosan61028@gmail.com
}

\begin{abstract}
In this article, we consider two types of multi-bit binary multipliers realizing the arithmetic multiplication of two positive numbers with a fixed point: a Vedic multiplier and a modified tree multiplier. Both structures are modeled for $(8 \times 8),(16 \times 16),(32 \times 32)$-bit binary multipliers in the Quartus II CAD environment based on the Altera EP2SGX30DF780C3 FPGAs of the Stratix-II-GX family. Their comparative analysis on hardware and time costs is performed. Based on the result of the comparisons, with the implementation of the $(32 \times 32)$-bit binary multiplier, the
\end{abstract}

multiplier proposed in this work gives a gain in speed up to $23 \%$ in comparison with the Vedic multiplier. In terms of hardware costs, the proposed multiplier reduces by $22 \%$ compared to the Vedic multiplier. In addition, the proposed structure can be scaled to a larger number of bits, for example $(64 \times 64),(128 \times 128),(256 \times 256)$ bits, and so on.

Keywords - binary multiplier; half-adder; full adder; parallel-prefix adder (PPA); a carry-save adder (CSA); Vedic multiplier (VM); ALUTs; delay time. 


\section{REFERENCES}

[1] Chervyakov N.I., Lyakhov P.A., Valueva M.V., Krivolapova O.V., Comparative analysis of the hardware implementation of the adder on FPGA // "Nauki. Innovation. Technologies »North-Caucasian Federal University. 2016. № 4. C. 98.

[2] Akash Kumar, Deepika Sharma. Analyze the performance of various types of adders for high-speed 32-bit multiplication and accumulation. 2013.-V.. 3. C. 1460.

[3] Prof. Rashmi Rahul Kulkarni. Comparison between different adder // Journal of IOSR VLSI and Signal Processing. 11-12, 2015. V 5. P. 2319-4197.

[4] SA Orlov., B. Ya. Zilker. Organization of computers and systems. Textbook for high schools. 2nd edition, 2011. pp. 192-206.

[5] Bhupender Pratap Singh., Rakesh Kumar. Design and Implementatin 8-bit Wallace Tree Multiplier. International Journal of Advanced Research in Electrical Electronics and
Instrumentation Engineering. Vol. 5, Issye 4, April 2016. P 2307-2311.

[6] Palaniappan Ramanathan., Ponnisamy Thangapandian Vanathi., Sundeepkumar Agarwal. High Speed Multiplier Design Using Decomposition Logic. Serbian journal of electrical engineering. Vol. 6, No. 1, May 2009, 33-42.

[7] Abhijeet Kumar., Dilip Kumar., Siddhi. Hardware Implementation of $16^{* 16}$ bit Multiplier and Square using Vedic Mathematics. International Conference on Signal, Image and Video Processing (ICSIVP) 2012. P. 309-314.

[8] Altera Corporation. Stratix II Device Handbook, Volume 1 2005// URL: http://www.ece.iastate.edu/ zambreno/classes/ cpre583/documents/altera/stx2_sii5v1_01.pdf. P. 20-22.

[9] David Harris. Taxonomy of parallel prefix networks. Harvey Mudd College. IEEE 2013 / P. 2213-2214.

[10] Reto Zimmermann. The architecture of the binary adder for the cell-based VLSI and their synthesis. Swiss Federal Institute of Technology, Zurich, 1997. pp. 34-39. 